\title{
Associação entre consumo de álcool no padrão binge e tabaco em estudantes de enfermagem
}

\author{
Bárbara de Oliveira Prado Sousa ${ }^{1}$, \\ André Luiz Thomaz de Souza², \\ Dival Cubas Junior ${ }^{3}$, \\ Gabriela Fulan e Silva ${ }^{4}$, \\ Manoel Antônio dos Santos ${ }^{5}$, \\ Sandra Cristina Pillon ${ }^{6}$
}

\section{RESUMO}

O estudo teve por objetivo avaliar o uso de álcool no padrão binge e a sua associação com o tabaco em estudantes de enfermagem. Estudo transversal desenvolvido com 182 estudantes. Foi utilizado formulário informações sociodemográficas, (AUDIT C) e (FTND). Dos participantes, 48,9\% foram classificados no padrão binge drinking e $6 \%$ eram fumantes. Constatou-se que o binge esteve associado à frequência de uso de álcool, a dose de consumo e a frequência de uso de cinco ou mais doses em uma única ocasião. Além disso, esse padrão de consumo associouse ao tempo de fumar após acordar, número de cigarros diários, consumo matutino, fumar em qualquer hora do dia, situações de proibição e casos de doença. A prática de binge esteve associada à frequência e à dose de consumo de álcool, assim como ao tabaco, porém, essa conclusão pode não retratar a realidade brasileira por se tratar de uma população específica.

Descritores: Estudantes de Enfermagem; Transtornos Relacionados ao Uso de Álcool; Bebedeira; Uso de Tabaco.

\footnotetext{
${ }^{1}$ Enfermeira, Doutora em Enfermagem Psiquiátrica. Ribeirão Preto, SP, Brasil. E-mail: barbaraprado89@hotmail.com.

2 Enfermeiro. Discente do Programa de Pós-Graduação em Enfermagem Fundamental, nível Doutorado, da Escola de Enfermagem de Ribeirão Preto da Universidade de São Paulo. Professor das Faculdades Integradas do Vale do Ribeira. Registro, SP, Brasil E-mail: alfenas2@hotmail.com.

${ }^{3}$ Acadêmico do curso de graduação em Enfermagem na Faculdades Integradas do Vale do Ribeira. Registro, SP, Brasil E-mail:

junior.cubas@hotmail.com.

${ }^{4}$ Enfermeira, Mestre em Enfermagem na Saúde do Adulto. Professora Titular das Faculdades Integradas do Vale do Ribeira. Registro, SP, Brasil Email: gabifurlan@yahoo.com.br.

${ }^{5}$ Psicólogo, Doutor em Psicologia Clínica. Professor Titular da Faculdade de Filosofia, Ciências e Letras de Ribeirão Preto da Universidade de São Paulo. Ribeirão Preto, SP, Brasil. E-mail: masantos@ffclrp.usp.br.

${ }^{6}$ Enfermeira, Doutora em Psiquiatria e Psicologia Médica. Professora Titular da Escola de Enfermagem de Ribeirão Preto da Universidade de São Paulo. Ribeirão Preto, SP, Brasil. E-mail: pillon@eerp.usp.br.
}

Artigo recebido: 09/08/2017.

Artigo aprovado: 18/04/2018.

Artigo publicado: 31/12/2018.

\section{Como citar esse artigo:}

Sousa BOP, Souza ALT, Cubas Junior D, Silva GF, Santos MA, Pillon SC. Associação entre consumo de álcool no padrão binge e tabaco em estudantes de enfermagem. Rev. Eletr. Enf. [Internet]. 2018 [acesso em:

];20:v20a36. Disponível em: https://doi.org/10.5216/ree.v20.48611. 


\section{INTRODUÇÃO}

O uso de bebidas alcoólicas e tabaco é um hábito comum no ambiente universitário em evidente crescimento. Muitas vezes esse hábito tem sido potencializado pelas experiências de sociabilidade dos jovens estudantes, que se sentem estimulados a consumir essas substâncias quando estão em contato com ambientes festivos. Também podem se sentir motivados em decorrência das transformações biopsicossociais por que passam em suas vidas, da facilidade no acesso ao uso e das dificuldades e atribulações inerentes à vida universitária. Esses fatores podem contribuir para aumentar os níveis de estresse e o uso de álcool e tabaco pode ser uma fonte de modulação e alívio imediato, e mesmo de enfrentamento para diversas situações ${ }^{(1)}$. No que tange ao consumo de álcool, o padrão binge drinking $(\mathrm{BD})$ entre estudantes universitários tem sido relatado de forma recorrente na literatura ${ }^{(2-4)}$.

O consumo de álcool no padrão binge pode ser definido como um uso pesado em um único episódio, até que as concentrações de álcool no sangue atinjam $0,08 \mathrm{~g} / \mathrm{dL}^{(3-5)}$. No indivíduo adulto, o padrão binge equivale ao consumo de cinco ou mais doses para homens e quatro ou mais doses para mulheres, ingeridas no prazo de duas horas $^{(6)}$.

Além do consumo de álcool, o uso de tabaco também é considerado um fator de risco para agravo à saúde. Seu uso no contexto universitário tem sido investigado com frequência em estudos internacionais ${ }^{(7)}$, sendo que a prática do tabagismo muitas vezes está associada ao uso de álcool. Entre os estudantes de enfermagem foi constatada uma prevalência no consumo de tabaco de $24 \%$ e de álcool de $82 \%$ em uma amostra de 237 participantes; ao relacionar o consumo de álcool e tabaco, identificou-se que $98 \%$ dos acadêmicos que fumavam também consumiam álcool em quantidades elevadas ${ }^{(8)}$.

Estudo realizado nos Estados Unidos evidenciou que a disseminação do uso do tabaco entre universitários ocorreu em conjunto com o uso intenso de álcool ${ }^{(7)}$. Esse dado sugere que o uso associado das duas substâncias pode ser decorrente da facilidade de estabelecer interação social, manter-se desperto e acalmar o estudante ao se sentir embriagado, principalmente em festas universitárias em que jovens buscam obter essas vantagens.

Entretanto, segundo levantamento nacional com universitários de 27 capitais brasileiras, os estudantes que consomem no padrão binge estão frequentemente expostos aos riscos imediatos inerentes a esse padrão de consumo, especialmente o envolvimento em acidentes de trânsito, sexo desprotegido, problemas com a lei, intoxicação alcoólica aguda, dificuldades acadêmicas, violência e abuso sexual sob influência do álcool(9).

Estudantes da área da saúde, principalmente os futuros enfermeiros, são considerados um grupo populacional de relevo e de especial interesse para o país, visto que são eles que irão transmitir conhecimentos sistematizados à comunidade e usar suas habilidades e competências profissionais para a implementação de práticas, hábitos e comportamentos saudáveis ${ }^{(10)}$. Nesse contexto, é necessário entender o consumo de tabaco e álcool nessa população, a fim de desenvolver estratégias de promoção de saúde e prevenção ao uso de drogas para melhoria das condições de saúde dos universitários. Desse modo, a instituição formadora estará investindo para minimizar problemas que afetam os setores social, econômico e político, e que estão vinculados ao consumo não recreacional de substâncias psicoativas.

Estudos brasileiros indicam que as evidências atualmente disponíveis sobre o uso de substâncias psicoativas estão voltadas, principalmente, para o conhecimento da frequência de consumo e associação com dados 
sociodemográficos ${ }^{(11)}$, análises de diferenças entre os $\operatorname{sexos}^{(12)}$ e abuso de medicamentos sem prescrição médica $^{(13)}$.

Embora os estudos epidemiológicos tenham avaliado o uso de álcool no padrão binge e o consumo de tabaco isoladamente ${ }^{(14-15)}$, no contexto brasileiro ainda são escassas as evidências sobre a associação entre as duas substâncias, principalmente no que se refere aos estudantes de enfermagem. Observa-se ainda que o comportamento em binge é mais comum em homens, entretanto, esse comportamento está em evidente crescimento em mulheres, o que necessita ser melhor explorado por ser um fenômeno recente e ainda pouco estudado nesse público.

Desse modo, ao considerar a relevância do tema e a necessidade de prover evidências consistentes, este estudo teve por objetivo avaliar o uso de álcool no padrão binge e sua associação com o consumo de tabaco em estudantes de enfermagem.

\section{MÉTODO}

Trata-se de um estudo transversal, descritivo, de abordagem quantitativa, desenvolvido com estudantes de enfermagem de uma Instituição de Ensino Superior localizada no Vale do Ribeira, litoral sul do Estado de São Paulo. O curso de enfermagem funciona em regime semestral, com tempo total de 10 semestres letivos (cinco anos).

Considerando o total de 261 (100\%) estudantes matriculados em todos os semestres letivos do curso, por meio de cálculo amostral (95\% de confiabilidade e 2\% de precisão), a amostra mínima necessária era de 156 estudantes. Os acadêmicos foram selecionados de acordo com os critérios de elegibilidade: estar matriculado regularmente no curso, de ambos os sexos, com idade acima de 18 anos e estar presente em sala de aula no momento da coleta de dados.

Ao final, participaram voluntariamente do estudo $182(69,7 \%)$ estudantes. A coleta de dados ocorreu no período de agosto a novembro de 2016, em sala de aula, após anuência da coordenação do curso, por meio de um questionário contendo: (1) Formulário de informação sociodemográfica; (2) Teste de Identificação do Uso de Álcool - C (AUDIT-C); (3) Teste de Dependência à Nicotina de Fagerström (FTND).

(1) Formulário de informações sociodemográficas: composto por sexo, faixa etária, renda mensal, ano em curso, estado civil, ter filhos, com quem reside, trabalho e religião.

(2) Teste de Identificação do Uso de Álcool - Consumo (AUDIT-C): trata-se de uma versão abreviada do AUDIT. Este instrumento apresenta três itens referentes à quantidade, frequência e consumo no padrão binge drinking ${ }^{(16)}$. Para a interpretação do consumo é necessário somar as respostas, que variam de zero a 12 pontos. A classificação final se diferencia em relação ao sexo, sendo: homens (cinco pontos) e mulheres (quatro pontos). No presente estudo foi utilizado como variável dependente somente o terceiro item deste instrumento, o qual caracteriza o consumo em binge. As demais variáveis do instrumento foram utilizadas para associação com esse padrão de consumo. Estudo mostrou níveis muito bons de sensibilidade $(0,90)$ e especificidade $(0,91)$ do instrumento. Os autores apresentaram as fragilidades e potencialidades da utilização do AUDIT em amostras de estudantes universitários e do público feminino ${ }^{(17)}$. Além disso, o AUDIT-C foi comparado com o AUDIT em sua versão original e também com o 
AUDIT 3, de modo que o AUDIT-C apresentou maior eficácia e melhores resultados quando comparado com as demais versões ${ }^{(17)}$.

(3) Teste de Dependência à Nicotina de Fagerström (FTND): este instrumento é utilizado para avaliar os níveis de dependência da nicotina ${ }^{(18)}$. O FTND foi adaptado para ser utilizado na população brasileira, sendo constituído por seis itens relativos a hábitos e comportamentos relacionados ao uso do tabaco. Sua classificação ocorre em cinco níveis de acordo com a pontuação: 0-2 muito baixo, 3-4 baixo, 5 médio, 6-7 elevado, 8-10 muito elevado.

Para avaliar a compreensão dos itens do instrumento foi realizado um teste piloto com 10 estudantes matriculados em diferentes semestres letivos, que não fizeram parte da amostra. 0 tempo despendido para o preenchimento do instrumento foi de, aproximadamente, 20 minutos. Ao término da aplicação dos questionários, todos foram guardados em envelopes lacrados sem identificação, a fim de garantir o anonimato dos respondentes.

O estudo foi aprovado pelo Comitê de Ética em Pesquisa envolvendo seres humanos sob o Parecer no 1.589.998 (CAAE: 56601316.4.0000.5490) da União das Instituições de Serviço, Ensino e Pesquisa - UNISEPE e conduzido conforme a resolução 466/2012 do Conselho Nacional de Saúde. Todos os participantes assinaram o Termo de Consentimento Livre Esclarecido - TCLE.

Os dados obtidos foram inseridos em um banco de dados com dupla digitação e as análises estatísticas realizadas por meio do programa Statistical Package for Social Science (SPSS) versão 19.0. Na análise descritiva foi realizada por meio do cálculo de frequência, média e desvio-padrão. O Teste Exato de Fisher e o Teste Quiquadrado $\left(\chi^{2}\right)$ foram aplicados para mensurar a associação entre o binge e as variáveis sociodemográficas, o AUDIT-C e o FTND. Para todos os testes foi considerado um nível de significância de $5 \%$ a partir de um intervalo de confiança (IC) de $95 \%$.

\section{RESULTADOS}

A amostra foi composta por 182 estudantes de enfermagem, predominantemente do sexo feminino 137 (75,3\%), com média de idade de 26,6 anos (DP=8,0), do 4ㅇa ano 45 (24,7\%), solteiros 105 (57,7\%), sem filhos 115 $(63,2 \%)$, que trabalhavam 126 (69,2\%). Além disso, eles possuíam renda salarial média de $R \$ 1.203,13$, sendo que $90,7 \%$ residiam com seus familiares. Pode-se observar que aproximadamente a metade dos estudantes consumia álcool no padrão binge (48,9\%). Além disso, o consumo de álcool no padrão binge foi prevalecente entre estudantes de religião católica e que se declararam praticantes (Tabela 1). 
Tabela 1: Informações sociodemográficas e BD entre estudantes de enfermagem (N=182). Registro, SP, Brasil, 2016.

\begin{tabular}{|c|c|c|c|c|c|c|c|}
\hline & & \multicolumn{6}{|c|}{ BD } \\
\hline & & \multicolumn{2}{|c|}{$\operatorname{Sim}(n=89)$} & \multicolumn{2}{|c|}{ Não (n=93) } & \multirow{2}{*}{$\begin{array}{c}\text { Teste } \\
\chi^{2}\end{array}$} & \multirow{2}{*}{ Valor de $p$} \\
\hline & & $n$ & $\%$ & $\mathrm{n}$ & $\%$ & & \\
\hline \multirow{3}{*}{ Sexo } & Feminino & 65 & 73,0 & 72 & 77,4 & \multirow{3}{*}{0,470} & \multirow{3}{*}{$0,30^{a}$} \\
\hline & Masculino & 24 & 27,0 & 21 & 22,6 & & \\
\hline & 17 a 29 anos & 55 & 61,8 & 65 & 69,9 & & \\
\hline \multirow[t]{2}{*}{ Faixa etária } & 30 a 40 anos & 29 & 32,6 & 21 & 22,6 & \multirow[t]{2}{*}{2,360} & \multirow[t]{2}{*}{$0,30^{b}$} \\
\hline & 41 a 57 anos & 5 & 5,6 & 7 & 7,5 & & \\
\hline \multirow{2}{*}{ Ano em curso } & $1^{\circ}$ e $2^{\circ}$ ano & 39 & 43,8 & 41 & 44,1 & \multirow{2}{*}{0,001} & \multirow{2}{*}{$0,54^{a}$} \\
\hline & $3^{\circ}, 4^{\circ}$ e $5^{\circ}$ ano & 50 & 56,2 & 52 & 55,9 & & \\
\hline \multirow{2}{*}{ Estado civil } & Solteiro & 49 & 55,1 & 56 & 60,2 & \multirow{2}{*}{0,496} & \multirow{2}{*}{$0,29^{a}$} \\
\hline & União consensual & 40 & 44,9 & 37 & 39,8 & & \\
\hline \multirow{2}{*}{ Com quem reside } & Sozinho & 11 & 12,4 & 6 & 6,5 & \multirow{2}{*}{1,874} & \multirow{2}{*}{$0,13^{a}$} \\
\hline & Familiares & 78 & 87,6 & 87 & 93,5 & & \\
\hline \multirow{2}{*}{ Religião } & Católico & 49 & 55,1 & 29 & 31,2 & \multirow{2}{*}{10,584} & \multirow{2}{*}{$<0,01^{a}$} \\
\hline & Não católico & 40 & 44,9 & 64 & 68,8 & & \\
\hline \multirow{2}{*}{ Praticante da religião } & Praticante & 45 & 50,6 & 62 & 66,7 & \multirow{2}{*}{4,869} & \multirow{2}{*}{$0,02^{\mathrm{a}}$} \\
\hline & Não praticante & 44 & 49,4 & 31 & 33,3 & & \\
\hline
\end{tabular}

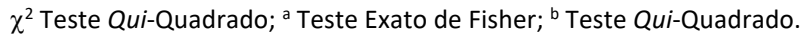

Os estudantes que consumiram álcool no padrão binge apresentaram maior frequência de consumo de bebidas alcoólicas (duas a quatro vezes por mês), além de ingerirem oito ou mais doses em um dia típico. Além disso, o consumo de cinco ou mais doses em uma única ocasião ocorreu na frequência de uma vez por mês (Tabela 2).

Tabela 2: Avaliação do BD e o padrão de consumo de álcool nos últimos 12 meses (AUDIT-C) entre estudantes de enfermagem ( $\mathrm{N}=182)$. Registro, SP, Brasil, 2016.

\begin{tabular}{|c|c|c|c|c|c|c|c|}
\hline & & \multicolumn{6}{|c|}{ BD } \\
\hline & & \multicolumn{2}{|c|}{$\operatorname{Sim}(n=89)$} & \multicolumn{2}{|c|}{ Não (n=93) } & \multirow{2}{*}{$\frac{\text { Teste }}{\chi^{2}}$} & \multirow{2}{*}{$\begin{array}{l}\text { Valor } \\
\text { de } p\end{array}$} \\
\hline & & 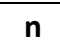 & $\%$ & $n$ & $\%$ & & \\
\hline \multirow{5}{*}{ Frequência de consumo } & Nunca & 2 & 2,2 & 77 & 82,8 & & \\
\hline & Uma vez por mês ou menos & 29 & 32,6 & 13 & 14,0 & & \\
\hline & $2-4$ vezes por mês & 32 & 36,0 & 3 & 3,2 & 127,300 & $<0,01$ \\
\hline & $2-3$ vezes por semana & 10 & 11,2 & - & - & & \\
\hline & 4 ou mais vezes por semana & 16 & 18,0 & - & - & & \\
\hline \multirow{4}{*}{$\begin{array}{l}\text { Doses de consumo em um dia } \\
\text { normal }\end{array}$} & $0-1$ & 19 & 21,3 & 88 & 94,6 & & \\
\hline & $2-3$ & 21 & 23,6 & 3 & 3,2 & & \\
\hline & $4-5$ & 18 & 20,2 & - & - & 13,424 & $<0,01$ \\
\hline & $6-7$ & 5 & 5,6 & - & - & & \\
\hline & 8 ou mais & 26 & 29,2 & 2 & 2,2 & & \\
\hline & Abstinentes & - & - & 93 & 100,0 & & \\
\hline Frequência de consumo de cinco & Menos que uma vez por mês & 24 & 27,0 & - & - & & \\
\hline ou mais doses em uma única & Uma vez por mês & 28 & 31,5 & - & - & 182,000 & $<0,01$ \\
\hline \multirow[t]{2}{*}{ ocasião } & Uma vez por semana & 11 & 12,4 & - & - & & \\
\hline & Quase todos os dias & 26 & 29,2 & - & - & & \\
\hline
\end{tabular}

$\chi^{2}$ Teste Qui-Quadrado.

Ao avaliar o consumo de tabaco (FTND), observou-se que apenas $6 \%$ dos estudantes eram fumantes. Nessa população, o BD foi associado ao hábito de fumar pela manhã (31 a 60 minutos após acordar), apresentavam 
dificuldades em não fumar em lugares proibidos, e que o cigarro do dia que trazia mais satisfação poderia ser fumado a qualquer hora do dia. Além disso, fumar entre 11 a 20 cigarros por dia, não fumar frequentemente pela manhã e fumar mesmo quando estivesse doente foram associados ao BD (Tabela 3).

Tabela 3: Comparação do padrão de consumo de tabaco (FTND) e BD entre estudantes de enfermagem ( $N=182$ ). Registro, SP, Brasil, 2016.

\begin{tabular}{|c|c|c|c|c|c|}
\hline \multirow{2}{*}{ Tabaco } & & \multicolumn{4}{|c|}{ BD (sim) } \\
\hline & & $\mathbf{n}$ & $\%$ & Teste $\chi^{2}$ & Valor de $p$ \\
\hline \multirow{4}{*}{ Em quanto tempo após acordar fuma o primeiro cigarro } & Abstêmios & 78 & 87,6 & \multirow{3}{*}{12,234} & \multirow{3}{*}{$<0,01^{b}$} \\
\hline & 31 a 60 minutos & 8 & 9,0 & & \\
\hline & De 6 a 30 minutos & 3 & 3,4 & & \\
\hline & Abstêmios & 79 & 88,8 & \multirow{3}{*}{8,476} & \multirow{3}{*}{$0,01^{\mathrm{b}}$} \\
\hline \multirow[t]{2}{*}{ Dificuldade em não fumar em lugares proibidos } & Não & 4 & 4,5 & & \\
\hline & Sim & 6 & 6,7 & & \\
\hline \multirow{3}{*}{ Cigarro do dia que gera maior satisfação } & Abstêmios & 78 & 87,6 & \multirow{2}{*}{12,234} & \multirow{2}{*}{$<0,01^{\mathrm{a}}$} \\
\hline & Qualquer cigarro do dia & 11 & 12,4 & & \\
\hline & Nenhum & 78 & 87,6 & \multirow{4}{*}{12,234} & \multirow{4}{*}{$<0,01^{b}$} \\
\hline \multirow{4}{*}{ Quantos cigarros fuma por dia } & Menos de 10 & 3 & 3,4 & & \\
\hline & De 11 a 20 & 6 & 6,7 & & \\
\hline & De 21 a 30 & 2 & 2,2 & & \\
\hline & Abstêmios & 78 & 87,6 & \multirow{3}{*}{12,234} & \multirow{3}{*}{$<0,01^{\mathrm{b}}$} \\
\hline \multirow[t]{3}{*}{ Fuma mais frequentemente pela manhã } & Não & 7 & 7,9 & & \\
\hline & Sim & 4 & 4,5 & & \\
\hline & Abstêmios & 78 & 87,6 & \multirow{3}{*}{12,141} & \multirow{3}{*}{$<0,01^{b}$} \\
\hline \multirow[t]{2}{*}{ Fuma mesmo doente } & Sim & 5 & 5,6 & & \\
\hline & Não & 6 & 6,7 & & \\
\hline
\end{tabular}

$\chi^{2}$ Teste Qui-Quadrado; ${ }^{\text {a }}$ Teste Exato de Fisher; ${ }^{\mathrm{b}}$ Teste Qui-Quadrado.

Ao comparar o binge drinking e o uso de cigarro, o BD foi prevalecente entre os fumantes $(11,1 \%$ versus 1,1\%). Entretanto, entre os fumantes, o BD foi predominante entre os estudantes com níveis baixo e médio de consumo $(p<0,01)$, dados não apresentados em tabela.

\section{DISCUSSÃO}

Os resultados encontrados indicam que há um expressivo número de estudantes engajados no consumo de álcool no padrão binge independentemente do sexo, com expressão significativa em relação à frequência, número de doses ingeridas e índices elevados (Tabela 2). Ao investigar a relação com o consumo de tabaco observa-se que, apesar da frequência reduzida (6\%), manteve associações significativas com o beber em binge.

$\mathrm{O}$ uso de bebidas alcoólicas e tabaco é uma prática comum entre universitários ${ }^{(2-3,8)}$. Estudos nacionais envolvendo esse comportamento entre estudantes de enfermagem ainda são incipientes, contudo, revelam um consumo nocivo do álcool(14) e tabaco(15). A adoção desse comportamento representa um desafio para a saúde pública, pois impacta negativamente as relações sociais ${ }^{(19)}$, diminui a resistência imunológica e eleva a propensão ao desenvolvimento de doenças cardiovasculares e hepáticas( ${ }^{(6)}$, além de favorecer o envolvimento em comportamentos de risco.

O uso do AUDIT-C em pesquisa envolvendo universitários mostrou que, dentre 163 estudantes de enfermagem, quase a metade consumia em níveis de risco e $19 \%$ fazia uso nocivo de álcool ${ }^{(14)}$. Estudo sobre o 
consumo de tabaco em estudantes de enfermagem revelou que, dentre 344 sujeitos, $83,6 \%$ nunca fumaram ${ }^{(20)}$. 0 consumo de álcool e tabaco pode ser considerado para os universitários como um "pacote de uso" socialmente aceitável, principalmente em festas universitárias e outros ambientes de descontração, favorecedores de oportunidades de interação social(7).

Por outro lado, a investigação sobre o uso de álcool e tabaco no contexto universitário possibilita identificar e compreender como essas duas substâncias se relaciona e potencializa seus efeitos nesta população. Cabe destacar que a Diretriz Curricular Nacional que norteia a formação em enfermagem preconiza que "o enfermeiro deve ser competente no cuidar da própria saúde física e mental e buscar seu bem-estar como cidadão"(21). Contudo, ao ser negligente no seu autocuidado, o acadêmico/profissional da saúde pode incentivar a comunidade na qual está inserido a assumir comportamentos que gerem riscos à saúde.

A identificação precoce de problemas associados ao uso do álcool e tabaco é um processo que envolve estratégias que combinam métodos de intervenção relativamente breves e simples, que podem ser implementados pelos profissionais inseridos na formação universitária. A literatura disponibiliza ferramentas que possibilitam a triagem relacionada ao consumo de álcool e tabaco. Dentre elas, destacam-se os seguintes instrumentos padronizados e aferidos para a população brasileira: AUDIT ${ }^{(16)}$, FTND $^{(18)}$ e o Alcohol, Smoking and Substance Involvement Screening - ASSIST ${ }^{(22)}$.

No presente estudo o BD foi prevalente em estudantes fumantes (dados descritivos) e que foram classificados no consumo baixo e médio de tabaco. $\mathrm{O}$ uso combinado de bebida alcoólica e tabaco é reconhecido por serem dois comportamentos fortemente relacionados entre $\mathrm{si}^{(23)}$ e que podem potencializar-se mutuamente. Esse consumo ocorre, principalmente, em situações que envolvem o BD. Evidências sugerem que o uso do tabaco, mesmo em fumantes leves, pode aumentar rapidamente após o beber em binge $e^{(24)}$, ou seja, quanto maior o consumo alcoólico (frequência e dose), maior o desejo de fumar.

O consumo de álcool entre estudantes universitários ainda representa um obstáculo a ser superado, principalmente quando se trata do uso no padrão binge. Provavelmente esse consumo está relacionado a um ambiente que oportuniza o convívio social sem a supervisão familiar, o que torna os estudantes vulneráveis ao uso de drogas de abuso ${ }^{(25)}$. É importante destacar que o consumo de álcool entre universitários é uma prática frequente, sendo a droga favorita dos estudantes. Muitas vezes eles se apoiam em uma falsa crença de que serão capazes de controlar os riscos vinculados ao uso do álcool(25). Tal situação em longo prazo pode culminar no uso problemático com impacto na vida profissional, pessoal e social.

Cabe destacar que as transformações ocorridas na sociedade nas últimas décadas modificaram o olhar que se lança sobre o comportamento de fumar, pois o que antes era visto de forma permissiva como um hábito atraente e glamoroso, hoje é encarado como indesejado e desrespeitoso no meio social ${ }^{(20)}$. Além disso, a legislação brasileira que regula a restrição do fumo em ambientes coletivos e a conscientização sobre os prejuízos associados ao uso do tabaco impõem limitações ao uso dessa substância, o que tem contribuído para o declínio no consumo.

Outro achado importante refere-se à associação entre a prática do binge e as variáveis correspondentes ao consumo do tabaco. Observa-se que os estudantes de enfermagem que consumiam álcool nesse padrão tinham o hábito de fumar de 31 a 60 minutos após acordar, apresentavam dificuldades em não fumar em lugares proibidos e referiram que o cigarro que trazia maior satisfação poderia ser fumado a qualquer hora do dia. Além 
disso, fumar entre 11 a 20 cigarros por dia, não fumar frequentemente pela manhã e fumar mesmo quando estivesse doente foram associados ao BD (Tabela 3). É fundamental que, no contexto universitário, sejam estimuladas ações direcionadas à prevenção no consumo e à educação quanto aos prejuízos biopsicossociais que envolvem o uso dessas substâncias. Nesse contexto, novos estudos que envolvam a temática álcool/tabaco no contexto universitário são necessários para subsidiar evidências para a implementação de tais ações preventivas.

O presente estudo apresenta algumas limitações. Primeiramente, os resultados devem ser avaliados com cautela, pois envolve a avaliação de apenas uma amostra de estudantes de enfermagem de munícipio brasileiro de pequeno porte. Em segundo lugar, apesar dos estudos demonstrar níveis muito bons de sensibilidade e especificidade do instrumento AUDIT C utilizou-se a versão abreviada do instrumento original (AUDIT). Por fim, o estudo envolveu somente uma instituição superior particular localizada no Vale do Ribeira, restringindo assim a avaliação de outras realidades, quer sejam públicas ou privadas. Desse modo, sugerimos que outros estudos sejam desenvolvidos para investigar os diferentes aspectos relacionados ao consumo em binge e o uso de tabaco em outras localidades.

Por outro lado, este estudo oferece, como principal avanço, o detalhamento na investigação sobre o binômio BD/tabaco no Vale do Ribeira, uma região com baixo índice de desenvolvimento humano (IDH) que até então não tinha sido objeto de investigação dessa natureza. Além disso, a pesquisa contribui com dados originais para a investigação sobre o panorama do uso de álcool e tabaco em estudantes de enfermagem.

\section{CONCLUSÃO}

O consumo de álcool no padrão BD foi elevado, considerando a população investigada. Contudo, o BD esteve associado a uma baixa e média classificação de dependência de tabaco. Os dados deste estudo fornecem subsídios para a tomada de decisão em ações voltadas para a prevenção do consumo de álcool e tabaco no contexto universitário, com vistas a minimizar problemas decorrentes do uso dessas substâncias em localidades brasileiras com baixo IDH. Os resultados lançam luz sobre a formação profissional em enfermagem e encorajam o desenvolvimento precoce de estratégias direcionadas ao autocuidado, com vistas a preservar a saúde do estudante.

\section{REFERÊNCIAS}

1. Pillon SC, Corradi-Webster CM. Teste de identificação de problemas relacionados ao uso de álcool entre universitários. Rev. enferm. UERJ [Internet]. 2006 [cited 2018 Dec 31];14(3):325-32. Available from: http://www.facenf.uerj.br/v14n3/v14n3a01.pdf. 2. McBride NM, Barrett B, Moore KA, Schonfeld L. The role of positive alcohol expectancies in underage binge drinking among college students. J Am Coll Health [Internet]. 2014 [cited 2018 Dec 31];62(6):370-9. Available from:

https://doi.org/10.1080/07448481.2014.907297.

3. Nunes JM, Campolina LR, Vieira MA, Caldeira AP. Consumo de bebidas alcoólicas e prática do binge drinking entre acadêmicos da área da saúde. Arch Clin Psychiatry (São Paulo) [Internet]. 2012 [cited 2018 Dec 31];39(3):94-9. Available from: https://doi.org/10.1590/S0101-60832012000300005.

4. Bedendo A, Andrade A, Opaleye E, Noto A. Binge drinking: padrão associado ao risco de problemas do uso de álcool entre universitários. Rev Lat Am Enfermagem [Internet]. 2017 [cited 2018 Dec 31];25:e2925. Available from:

https://doi.org/10.1590/1518-8345.1891.2925.

5. Saraiva SS, Maia Filho ALM. Consumo de bebidas alcoólicas e prática do binge drinking entre acadêmicos de Odontologia de uma IES. Rev Bras Odontol [Internet]. 2015 [cited 2018 Dec 31];72(1-2):104-8. Available from:

http://revodonto.bvsalud.org/scielo.php?script=sci_arttext\&pid=S0034-72722015000100020\&lng=en\&nrm=iso. 
6. National Institute on Alcohol Abuse and Alcoholism. Drinking Levels Defined | National Institute on Alcohol Abuse and Alcoholism (NIAAA) [Internet]. Washington, DC: National Institutes of Health; [cited 2018 Dec 31]. Available from:

https://www.niaaa.nih.gov/alcohol-health/overview-alcohol-consumption/moderate-binge-drinking.

7. Nichter M, Nichter M, Carkoglu A, Lloyd-Richardson E. Smoking and drinking among college students: "It's a package deal." Drug Alcohol Depend [Internet]. 2010 [cited 2018 Dec 31];106(1):16-20. Available from:

https://doi.org/10.1016/i.drugalcdep.2009.07.025.

8. López-Maldonado MC, Luis MAV, Gherardi-Donato EC da S. Consumo de drogas lícitas en estudiantes de enfermería de una universidad privada en Bogotá, Colombia. Rev Lat Am Enfermagem [Internet]. 2011 [cited 2018 Dec 31];19(spe):707-13. Available from: https://doi.org/10.1590/S0104-11692011000700007.

9. Presidência da República, Secretaria Nacional de Políticas sobre Drogas. I Levantamento Nacional sobre o Uso de Álcool, Tabaco e Outras Drogas entre Universitários das 27 Capitais Brasileiras [Internet]. Brasília: SENAD, 2010 [cited 2018 Dec 31]. Available from: http://www.grea.org.br/userfiles/GREA-ILevantamentoNacionalUniversitarios.pdf.

10. Blandón JAP, Molina AA, Martín MR, Campos MDML (2017) Influence of Nurse Education on Healthy Lifestyle Habits of Students: A Literature Review. J Nurs Care [Internet]. 2017 [cited 2018 Dec 31];6:384. Available from:

https://doi.org/10.4172/2167-1168.1000384.

11. Silveira RE, Santos ÁS, Pereira GA. Consumo de álcool, tabaco e outras drogas entre adolescentes do ensino fundamental de um município brasileiro. Rev Enferm Ref [Internet]. 2014 [cited 2018 Dec 31];IV Série(No 2):51-60. Available from:

https://doi.org/10.12707/RIII12112.

12. Fachini A, Furtado EF. Uso de álcool e expectativas do beber entre universitários: uma análise das diferenças entre os sexos. Psicol Teor e Pesqui [Internet]. 2013 [cited 2018 Dec 31];29(4):421-8. Available from: https://doi.org/10.1590/S010237722013000400008 .

13. Ehigiator O, Azodo CC, Ehizele AO, Ezeja EB, Ehigiator L, Madukwe IU. Self-medication practices among dental, midwifery and nursing students. Eur J Gen Dent [Internet]. 2013 [cited 2018 Dec 31];2(1):54-7. Available from: https://doi.org/10.4103/2278$\underline{9626.106813 .}$.

14. Maciel MED, Vargas D. Consumo de álcool entre estudantes de enfermagem Alcohol consumption among nursing students. Rev Pesqui Cuid é Fundam Online [Internet]. 2017 [cited 2018 Dec 31];9(1):64-70. Available from: https://doi.org/10.9789/21755361.2017.v9i1.64-70.

15. Silva BP, Corradi-Webster CM, Donato ECSG, Hayashida M, Siqueira MM. Transtornos mentais comuns e consumo de bebida alcoólica e tabaco entre estudantes de enfermagem de uma universidade pública na Amazônia Ocidental brasileira. SMAD Rev Eletrônica Saúde Ment Álcool e Drog (Edição em Port) [Internet]. 2014 [cited 2018 Dec 31];10(2):93-100. Available from: https://doi.org/10.11606/issn.1806-6976.v10i2p93-100.

16. Bradley KA, DeBenedetti AF, Volk RJ, Williams EC, Frank D, Kivlahan DR. AUDIT-C as a Brief Screen for Alcohol Misuse in Primary Care. Alcohol Clin Exp Res [Internet]. 2007 [cited 2018 Dec 31];31(7):1208-17. Available from: https://doi.org/10.1111/j.15300277.2007.00403.x.

17. Meneses-Gaya C de, Zuardi AW, Loureiro SR, Crippa JAS. Alcohol Use Disorders Identification Test (AUDIT): An updated systematic review of psychometric properties. Psychol Neurosci [Internet]. 2009 [cited 2018 Dec 31];2(1):83-97. Available from: https://doi.org/10.3922/j.psns.2009.1.12.

18. Fagerstrom KO, Schneider NG. Measuring nicotine dependence: a review of the Fagerstrom Tolerance Questionnaire. J Behav Med [Internet]. 1989 [cited 2018 Dec 31];12(2):159-82. Available from:

https://link.springer.com/article/10.1007\%2FBF00846549?LI=true.

19. Moretti-Pires RO, Ferro SBG, Büchele F, Oliveira HM, Gonçalves MJF. Enfermeiro de Saúde da Família na Amazônia: conceitos e manejo na temática do uso de álcool. Rev Esc Enferm USP [Internet]. 2011 [cited 2018 Dec 31];45(4):926-32. Available from: https://doi.org/10.1590/S0080-62342011000400019.

20. Echer IC, Corrêa APA, Ferreira SAL, Lucena AF. Tabagismo em uma escola de enfermagem do sul do Brasil. Texto Context Enferm [Internet]. 2011 [cited 2018 Dec 31];20(1):152-9. Available from: https://doi.org/10.1590/S0104-07072011000100018. 21. Resolução CNE/CES no 3, de 7 de novembro de 2001 (BR). Institui Diretrizes Curriculares Nacionais do Curso de Graduação em Enfermagem [Internet]. Brasília, DF: Ministério da Educação, 2001 [cited 2018 Dec 31]. Available from:

http://portal.mec.gov.br/cne/arquivos/pdf/CESO3.pdf.

22. Henrique IFS, De Micheli D, Lacerda RB, Lacerda LA, Formigoni MLOS. Validação da versão brasileira do teste de triagem do envolvimento com álcool, cigarro e outras substâncias (ASSIST). Rev Assoc Med Bras [Internet]. 2004 [cited 2018 Dec 31];50(2):199206. Available from: https://doi.org/10.1590/S0104-42302004000200039.

23. Acosta MC, Eissenberg T, Nichter M, Nichter M, Balster RL. Characterizing early cigarette use episodes in novice smokers. Addict Behav [Internet]. 2008 [cited 2018 Dec 31];33(1):106-21. Available from: https://doi.org/10.1016/j.addbeh.2007.09.005.

24. King AC, Epstein AM. Alcohol dose-dependent increases in smoking urge in light smokers. Alcohol Clin Exp Res [Internet]. 2005 [cited 2018 Dec 31];29(4):547-52. Available from: https://doi.org/10.1097/01.ALC.0000158839.65251.FE. 
Sousa BOP, Souza ALT, Cubas Junior D, Silva GF, Santos MA, Pillon SC.

25. Cardoso FM, Barbosa HA, Costa FM, Vieira MA, Caldeira AP. Fatores associados à prática do binge drinking entre estudantes da área da saúde. Rev CEFAC [Internet]. 2015 [cited 2018 Dec 31];17(2):475-84. Available from: https://doi.org/10.1590/1982-

021620158914. 